\title{
Stability of a Fiber Optical Parametric Oscillator With and Without a Seed Signal
}

\author{
Ioannis Begleris and Peter Horak \\ Optoelectronics Research Centre, University of Southampton, Southampton, SO17 1BJ, UK \\ i.begleris@soton.ac.uk
}

\begin{abstract}
We present a numerical model of a unidirectional fiber optical parametric oscillator. Power fluctuations between signal round-trips are investigated and found to be significant. Finally, the option of seeding the signal wave is discussed.
\end{abstract}

OCIS codes: $060.2320,060.4370$

\section{Introduction}

Parametric amplification in optical fibers is an interesting option to generate waves at wavelengths where laser sources are not readily available. However, the process relies on phase matching and thus fiber non-uniformities significantly degrade the conversion efficiency in long fibers. One way to combat this effect is to use only short lengths of highly nonlinear fiber. To further enhance conversion efficiency, the generated signal wave can be recirculated in the fiber thus forming a fiber optical parametric oscillator (FOPO). Here we present a numerical model of a FOPO based on the nonlinear Schrödinger equation to capture the full nonlinear spectral and temporal dynamics on timescales not generally accessible in experiments. The stability of the output with respect to pump wavelength and power is discussed. Finally, a seeded FOPO is considered.

a)

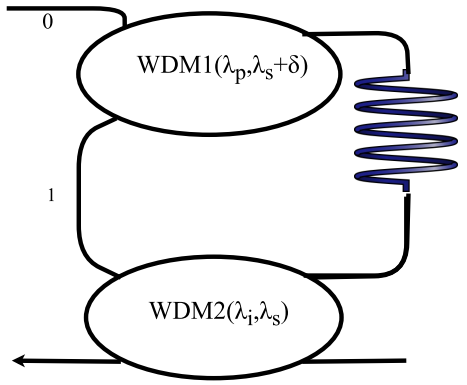

b)

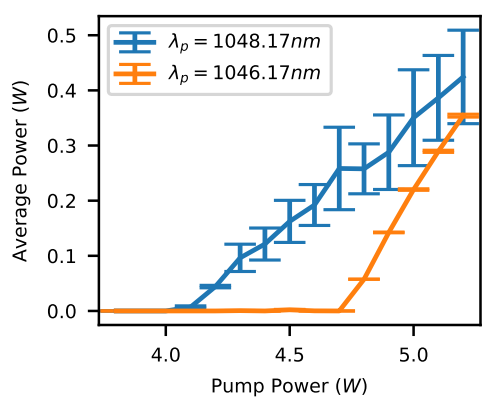

c)

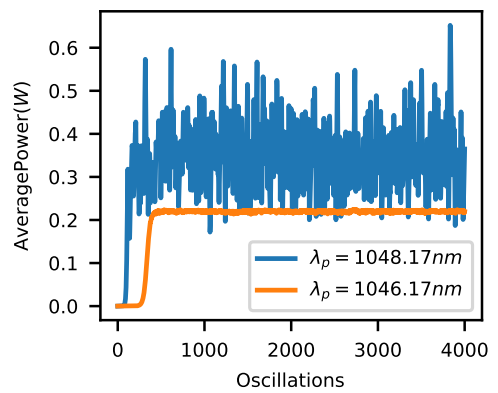

Fig. 1: (a) Schematic diagram of the fiber oscillator. (b) Output idler power versus input power for two pump wavelengths $\lambda_{p 1}, \lambda_{p 2}$. (c) Power versus round trip for a pump power of $5 \mathrm{~W}$.

\section{Methods}

Figure 1a shows a schematic of the FOPO [1]. Two wavelength division multiplexers (WDMs) WDM1 $\left(\lambda_{p}, \lambda_{s}+\delta\right)$, $\operatorname{WDM} 2\left(\lambda_{i}, \lambda_{s}\right)$ are used as input/output couplers, respectively, where the first argument is the transmitted wavelength and the second is the ejected wavelength and the subindices are for pump, signal, and idler respectively. An offset $\delta$ is allowed for in WDM1, and a further cumulated round trip loss is applied at position 1 . The fiber considered is an $18 \mathrm{~m}$ LMA-PM-5 photonic crystal fiber by NKT Photonics [2]. In the numerical implementation, the WDMs are modeled using the scattering matrix representation [3] and light propagation in the fiber is simulated by solving the generalized nonlinear Schrödinger equation using the split step Fourier method [4].

An oscillation within the FOPO begins with the input of the pump wave through WDM1 into the oscillator. It then propagates through the fiber where signal and idler sidebands are created. The idler (anti-Stokes) wave is extracted from the ring at WDM2 while the signal is retained and recombined at WDM1 with the pump for the next round trip. This process continues until an equilibrium between parametric gain and round trip loss is achieved. 


\section{Results}

First we study the system dynamics with respect to pump power and wavelength. Fig.1b shows the average output idler power as well as its variance between round trips (error bars) for two pump wavelengths $\lambda_{p 1}=1048.17 \mathrm{~nm}\left(\lambda_{s 1} \approx 1200\right.$ $\left.\mathrm{nm}, \lambda_{i 1} \approx 930 \mathrm{~nm}\right)$ and $\lambda_{p 2}=1046.17 \mathrm{~nm}\left(\lambda_{s 2} \approx 1230 \mathrm{~nm}, \lambda_{i 2} \approx 903 \mathrm{~nm}\right)$. The WDMs and cumulated loss are set up such that the maximum transmittance within the oscillator is at the signal wavelength $\lambda_{s 1}(\approx 72 \%)$. The FOPO is simulated for 4000 round trips and the output power is considered as the mean of the final 100 oscillations. The threshold at which the oscillator starts lasing is lower for $\lambda_{p 1}$ since the transmittance within the cavity is higher and the output idler is perfectly matched to WDM2. However, there are large power fluctuations between round trips. For $\lambda_{p 2}$ the lasing threshold is higher and the output power lower, but the power output is much more stable. This difference in behavior is seen even more clearly in the FOPO dynamics versus round trip number, Fig. 1c. We attribute the power fluctuations at $\lambda_{p 1}$ to significant spectral broadening observed in the simulated signal spectra and the generation of cascaded four-wave mixing processes that create highly nonlinear and unstable dynamics.
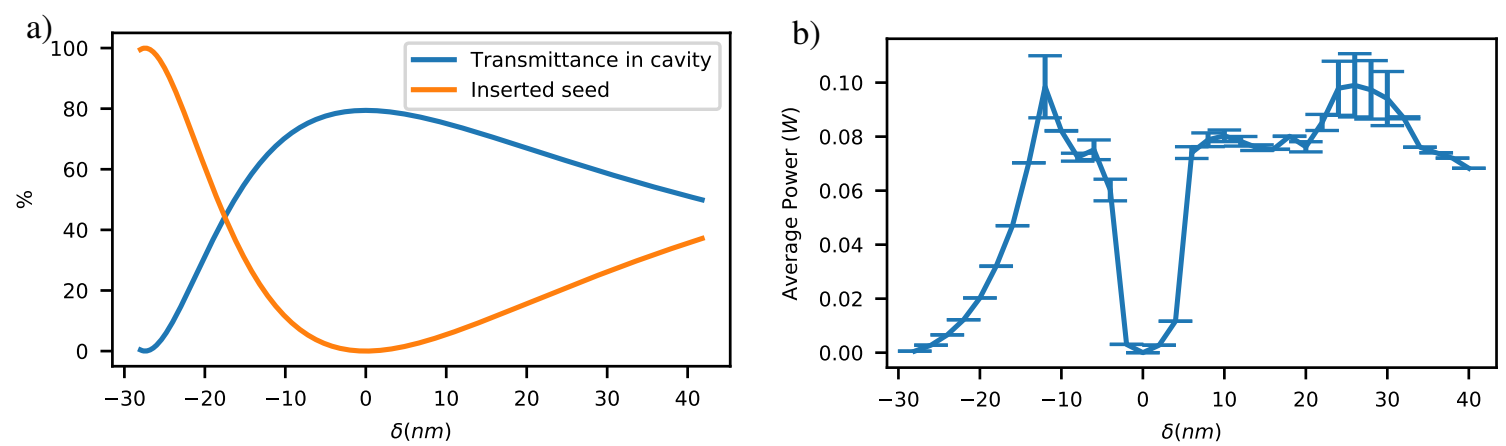

Fig. 2: a) Percentage of the inserted seed and its transmission within the cavity and (b) FOPO output power versus WDM1 offset $\delta$.

In order to reduce the power fluctuations, we next consider seeding the signal in the oscillator. For the example shown in Fig. 2 we assume a pump wavelength $\lambda_{p 3}=1051.5 \mathrm{~nm}$, pump power $P_{p 3}=2 \mathrm{~W}$, seed signal wavelength $\lambda_{s 3}=$ $1095 \mathrm{~nm}$, and seed power $P_{s 3}=100 \mathrm{~mW}$, which generates an idler at $\lambda_{i}=1011.4 \mathrm{~nm}$. In this seeded configuration, WDM1 needs to be detuned by the offset $\delta$ from resonance with the signal wavelength such that a fraction of the signal power can enter the oscillator, which on the other hand also increases the round trip signal loss, Fig. 2a. For these parameters and without the seed, the FOPO would operate below its threshold. The seed therefore acts to bring the FOPO to lasing and to define specific signal and idler operating wavelengths. The corresponding FOPO output power is shown in Fig. 2b. For $\delta=-30 \mathrm{~nm}$, all of the seed is inserted into the cavity but likewise the signal inside the cavity is ejected. Thus no signal can circulate and the FOPO operates below threshold. As $\delta$ increases, so does the output of the oscillator until a maximum is reached at $\delta \approx-10 \mathrm{~nm}$. For $\delta \approx 0$ no idler power is generated again because no seed is being inserted into the cavity. For $\delta>0$ the output power increases again since there is enough signal transmitted within the cavity and seed inserted into the system. However, above a certain power threshold we again find larger output power fluctuations, similar to the unseeded configuration, indicating that additional nonlinear effects start to generate cascade waves that introduce an instability in the system.

In conclusion, both in the single pump and seeded configurations a clear instability is exhibited at large cavity powers. The timescales at which this instability is simulated is small in comparison to what can be experimentally measured. However this power fluctuation needs to be taken into consideration in applications of FOPOs.

This work was supported by the EPSRC CDT in Next Generation Computational Modelling (grant EP/L015382/1).

\section{References}

1. E. A. Zlobina, S. I. Kablukov, S. A. Babin and A. Sergey, "High-efficiency CW all-fiber parametric oscillator tunable in $0.92-1 \mu \mathrm{m}$ range," Opt. Express 23, 833-838 (2015).

2. E. A. Zlobina, S. I. Kablukov and S. A.Babin, "Phase matching for parametric generation in polarization maintaining photonic crystal fiber pumped by tunable Yb-doped fiber laser," J. Opt. Soc. Am. B 29, 1959-1967 (2012).

3. G. Keiser, Optical Fiber Communications (McGraw-Hill Higher Education, 1991).

4. G. P. Agrawal, Nonlinear Fiber Optics (Academic Press, 2013). 